\title{
Large Eddy Simulation of Laminar and Turbulent Flow on Collocated and Staggered Grids
}

\author{
M. J. Ketabdari ${ }^{1}$ and H. Saghi ${ }^{2}$ \\ ${ }^{1}$ Faculty of Marine Technology, Amirkabir University of Technology, 424 Hafez Avenue, Tehran, P.O. Box 15875-4413, Iran \\ ${ }^{2}$ Department of Civil Engineering, Ferdowsi University, Mashhad, Iran \\ Correspondence should be addressed to M. J. Ketabdari, ketabdar@aut.ac.ir
}

Received 28 March 2011; Accepted 11 May 2011

Academic Editors: S. W. Chang, A. A. Kendoush, A. Postelnicu, and S. C. M. Yu

Copyright ( 2011 M. J. Ketabdari and H. Saghi. This is an open access article distributed under the Creative Commons Attribution License, which permits unrestricted use, distribution, and reproduction in any medium, provided the original work is properly cited.

\begin{abstract}
Momentum and Continuity are the basic equations for fluid flow modeling. The momentum equations in their final form are known as Navier-Stokes equations and can be solved using different numerical methods. There are several approaches such as SIMPLE, PISO, and Fractional Step for solving these equations. In solution procedure, it needs to decide where to store the velocity components. Staggered and Collocated grids can be used to evaluate this problem. On Staggered grids, the velocity components are stored at the cell face, and the scalar variables such as pressure are stored at the central nodes. However, on Collocated grids, all parameters are defined at the same location at the central nodes. The Staggered grids method gives more accurate pressure gradient estimation. However, Collocated grids method is simpler for solving the equations. In this paper, for solving NavierStokes equations, Collocated and Staggered grids are employed. Comparison of horizontal and vertical velocities and stream lines at various Reynolds numbers was performed. The results were validated using standard tests such as lid-driven cavity, channel and backward facing step. Discussion is made on accuracy of these methods to estimate horizontal and vertical velocity profiles.
\end{abstract}

\section{Introduction}

The fundamental equations of motion known as NavierStokes (N-S) equations are basically valid for laminar flow. To use them for turbulent flow, time, and spatial averaged form of these equations are used [1]. Different approaches such as Large Eddy Simulation (LES) and Direct Numerical Solution (DNS) models can be used to model turbulent flow. The classical models use the Reynolds Average Navier Stokes (RANS) equations. Complication of computational procedure for unsteady turbulent flow simulations is due to the very large number of grid points to be resolved for a wide range of length scales. In DNS, all scales are resolved both spatially and temporally. In LES approach, the equations are filtered to separate large eddies from small ones. In these models, it is assumed that the largest eddies interact strongly with the mean flow and contain most of the energy. Therefore, the main effects of turbulence can be modeled by this approach accurately [2].

There are several methods such as SIMPLE, PISO, and N-S equations. The SIMPLE algorithm was originally developed by Patankar and Spalding (1972) on the Staggered grids arrangement and involves one predictor step and one corrector step. While PISO method can be regarded as an extension of SIMPLE method with further correction steps [1]. The Fractional step method of Kim and Moin (1985) provides an approach that does not need to predict pressure in the predictor step. In this method, the N-S equations split into three equations containing convective, diffusive and pressure terms [3].

In this paper, a turbulent model was developed using Staggered and Collocated grids. To validate the model some standard tests such as lid-driven cavity, Channel flow and Backward-facing step were used.

Several researchers studied these tests. Prasad and Koseff [4] performed a series of experiments in lid-driven cavity for Reynolds number between 3200 and 10000 and spanwise aspect ratio (SAR) between $0.25: 1$ and $1: 1$. They showed that the flow is strongly affected by the SAR and Reynolds number so that this effect is different at low and high Reynolds numbers. Gokarn et al. [5] solved incompressible 
N-S equation using Large Eddy Simulation and partialstaggered variable arrangement. They validated the numerical scheme by simulating lid-driven cavity flow and flow over a Backward facing step. The results were compared with experimental data and demonstrated good agreement. Kim et al. [6] solved the unsteady N-S equation and DNS method at a Reynolds number of 3300 to simulate the fully developed channel flow. They compared the numerical results with the existing experimental data and discussion was made on origin of errors. Nie and Armaly [7] estimated the boundaries of the reverse flow regions in a threedimensional (3D) backward-facing step using Laser-Doppler velocity measurement as a function of the Reynolds numbe in a range of 100 to 8000 to cover the laminar, transitional and turbulent flow regimes. Their results well agreed with measurements of Alamry et al. [8] in the laminar and turbulent flow regimes.

\section{Governing Equations}

The governing equations of incompressible fluids which are Continuity and N-S equations can be expressed as

$$
\begin{gathered}
\frac{\partial u}{\partial x}+\frac{\partial v}{\partial y}=0 \\
\frac{\partial(u)}{\partial t}+\nabla \cdot(u V)=\frac{-1}{\rho} \frac{\partial P}{\partial x}+\nabla \cdot(\nu \nabla u)+S_{x}, \\
\frac{\partial(v)}{\partial t}+\nabla \cdot(v V)=\frac{-1}{\rho} \frac{\partial P}{\partial y}+\nabla \cdot(\nu \nabla v)+S_{Y},
\end{gathered}
$$

where $t$ is time, $V$ is the velocity vector, $u$ and $v$ are velocity components in $X$ and $Y$ direction, respectively, $P$ is the static pressure, $\nu$ is the kinematic viscosity, $\rho$ is the fluid density, $S_{\phi}$ is the source term including gravity acceleration in the $\phi$ direction [1].

In this research, LES model is used to simulate turbulent flow. It relies on the definition of large and small scales in which the former are solved and the later are modeled. Figure 1 shows the schematic view of this procedure.

To separate the large scales from the small ones, filtering process is used. A filtered variable, denoted by an over bar, is defined as

$$
\bar{f}(x)=\int f^{\prime}(x) G\left(x, x^{\prime}, \bar{\Delta}\right) d x^{\prime}
$$

where $D$ is the entire domain, $G$ is the filter function, and $\bar{\Delta}$ is the filter width, which is the wavelength of the smallest scale. The filter function determines the size and structure of the small scales. If the filtering operation is applied to the governing equation, the filtered equation of motion is obtained. For incompressible flow of a Newtonian fluid the conservation form can be expressed as

$$
\begin{gathered}
\frac{\partial \bar{u}_{i}}{\partial x_{i}}=0 \\
\frac{\partial \bar{u}_{i}}{\partial t}+\frac{\partial}{\partial x_{j}}\left(\bar{u}_{i} \bar{u}_{j}\right)=\frac{-1}{\rho} \frac{\partial \bar{p}}{\partial x_{i}}-\frac{\partial \tau_{i j}}{\partial x_{j}}+\nu \frac{\partial^{2} \bar{u}_{i}}{\partial x_{j} \partial x_{j}} .
\end{gathered}
$$

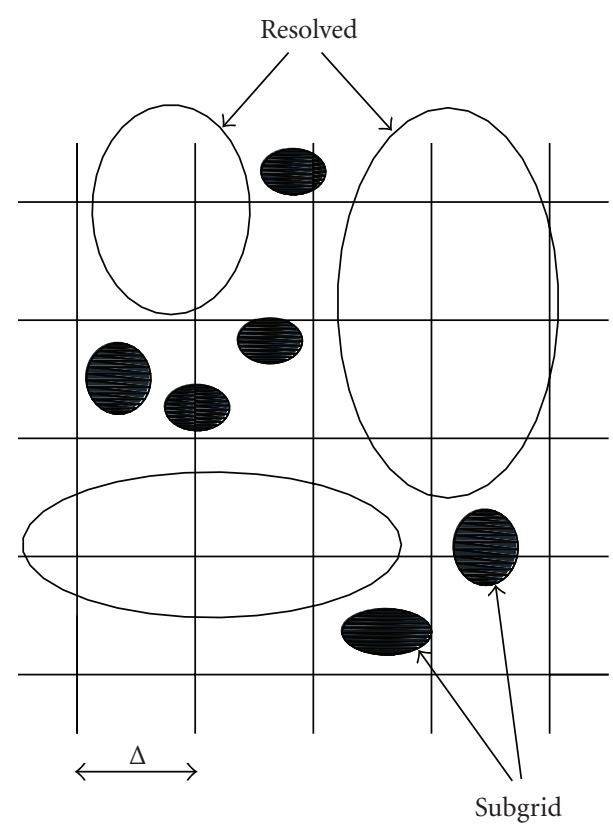

FIGURE 1: Schematic view of the simplest scale separation operator in LES model.

These equations just govern the evolution of the large scales. Therefore the effect of the small scales must be modeled through a subgrid-scale (SGS) stress term as

$$
\tau_{i j}=\overline{u_{i} u_{j}}-\bar{u}_{i} \bar{u}_{j}
$$

The Smagorinsky model is one of the various SGS models. This model can be expressed as [9]:

$$
\begin{gathered}
v_{t}(i, j)=\left(C_{S} \Delta\right)^{2}\left(2\left|\bar{S}_{i j}\right|^{2}\right)^{1 / 2}, \\
\bar{S}_{i j}=\frac{1}{2}\left(\frac{\partial \bar{u}_{j}}{\partial x_{i}}+\frac{\partial \bar{u}_{i}}{\partial x_{j}}\right), \\
\Delta=\left(\Delta_{x} \Delta_{y}\right)^{1 / 2},
\end{gathered}
$$

where $v_{t}$ is turbulence eddy viscosity, $C_{s}$ is a constant assumed to lie in the range of 0.065 to 0.25 [10], $\Delta$ is the length of filter, $\bar{S}_{i, j}$ is the strain rate tensor, $\bar{u}_{i}, \bar{u}_{j}$ are the filtered velocity in $X$ and $Y$ direction and $\Delta_{x}, \Delta_{y}$, and $\Delta_{z}$ are the mesh size in $X, Y$ and $Z$ direction, respectively.

Finally, the total kinematic viscosity is derived as

$$
v(i, j)=\frac{\mu}{\rho}+v_{t}(i, j)
$$

\section{Staggered and Collocated Grids}

The solution algorithm for the governing equations is consisting of discretization of flow domain and storing the velocity vectors. Two methods denoted as Collocated Grids (C.G) and Staggered Grids (S.G) are used in this procedure. On C.G, all velocity components and scalar variables such as pressure are stored in the same locations (see Figure 2). 


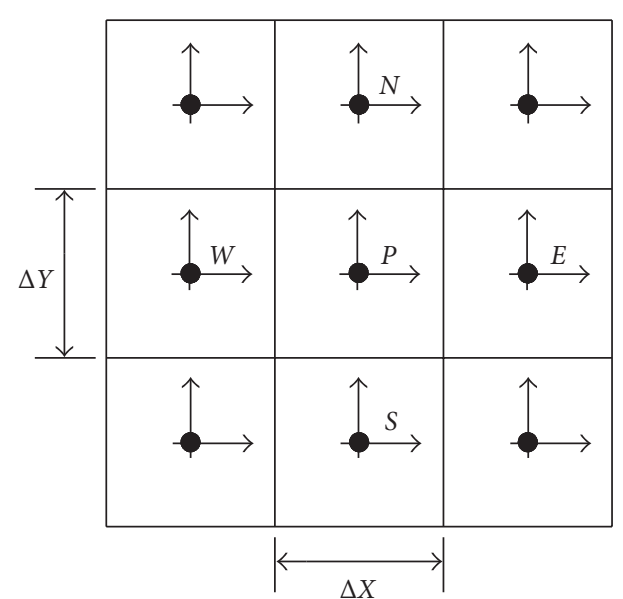

FIgure 2: Control volume in C.G.

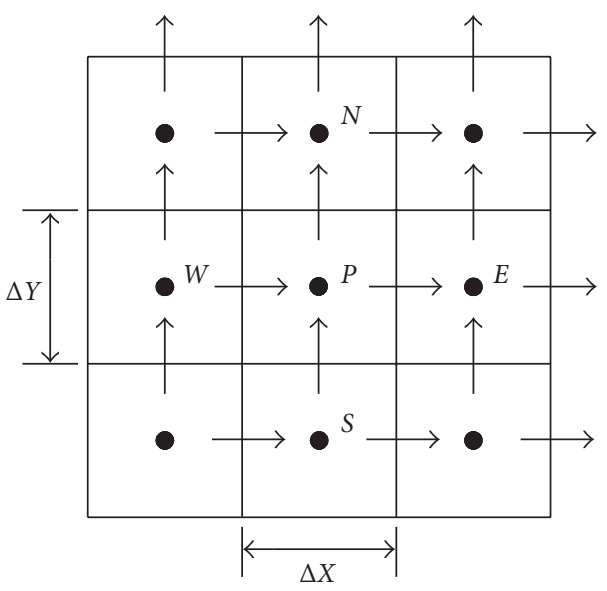

Figure 3: Control volume in a S.G.

Although application of C.G is simple, it cannot distinguish between a non-uniform pressures field and a uniform one. In S.G method, velocities are stored at the faces of control volume (see Figure 3).

One of the advantages of the S.G arrangement is that velocities are generated at locations where needed for the convection computations. While on C.G, stored velocities at the center of control volumes cannot be used directly. In this situation, for calculation of convection term, Rie-Chow interpolation is used.

\section{Solution Algorithm}

The solution algorithm employed in this study is a finite volume method based on the PISO predictor corrector algorithm coupled with a pressure correction to discretize the governing equations on structure grids.

In this method, the filtered N-S equations, are discretised using the finite volume method where the domain $D$ is divided into cells $\delta V_{i}$ so that $\sum \delta V_{i}=D$ and $\cap_{i}\left(\delta V_{i}\right)=$ $\phi$. Integration of the dependent variables over each cell together with application of Gauss' theorem generates a set of discretised equations. The two-dimensional N-S equations are discritized using Finite volume method as

$$
\begin{aligned}
& a_{P U} u_{P}=\sum a_{N B} u_{N B}-\frac{P(i+1, j)-P(i, j)}{d x}+S_{u}, \\
& a_{P V} v_{P}=\sum a_{N B} v_{N B}-\frac{P(i, j+1)-P(i, j)}{d y}+S_{v},
\end{aligned}
$$

where $u_{N B}$ and $v_{N B}$ are velocity components in $\mathrm{X}$ and $\mathrm{Y}$ direction and in neighboring cells $(N B=E, W, N, S)$ and $a_{N B}$ are the related coefficients needs to be estimated. There are several schemes such as upwind, central differencing and power law to estimate these coefficients.

In this research, to estimate the coefficients power law scheme is used as

$$
a_{i}=D_{i} * \operatorname{Max}\left[0,\left(1-0.1\left|P e_{i}\right|\right)^{5}\right]+\operatorname{Max}\left[F_{i}, 0\right],
$$

where $P_{e_{i}}, D_{i}$, and $F_{i}$ are the Peclet number, diffusion and convection terms on neighboring cells, respectively. The peclet number is the ratio of convection to diffusion terms. Theses terms are estimated on S.G as

$$
\begin{gathered}
D_{E}=D_{W}=\frac{\mu(i, j) d y}{d x}, \\
D_{N}=D_{S}=\frac{\mu(i, j) d x}{d y}, \\
F_{E}=F_{W}=\rho U d y, \\
F_{N}=F_{S}=\rho V d x .
\end{gathered}
$$

Diffusion terms are estimated on C.G similar to S.G. However, convection terms are estimated using interpolation techniques.

The discritized N-S equations must be solved for all control volumes to estimate the velocity components. But in this step, the unknown pressures in the control volumes should be predicted.

In next step, Momentum equation is solved using predicted pressure field and Three Diagonal Matrix Algorithm (TDMA) as

$$
\begin{gathered}
a_{P U} u_{P}=\sum a_{N B} u_{N B}-\frac{P^{*}(i+1, j)-P^{*}(i, j)}{d x}+S_{u}, \\
a_{P V} v_{P}=\sum a_{N B} v_{N B}-\frac{P^{*}(i, j+1)-P *(i, j)}{d y}+S_{v}, \\
P=P^{*}+P^{\prime},
\end{gathered}
$$

where $P^{*}, P$, and $P^{\prime}$ are the predicted, corrected and correction pressure, respectively.

In next step, the pressure correction equation is derived as

$$
\begin{gathered}
a_{P P} P_{P}^{\prime}=a_{E P} P_{E}^{\prime}+a_{W P} P_{W}^{\prime}+a_{S P} P_{S}^{\prime}+a_{N P} P_{N}^{\prime}, \\
a_{E P}=a_{W P}=\rho d y, \\
a_{S P}=a_{N P}=\rho d x, \\
a_{P P}=a_{E P}+a_{W P}+a_{S P}+a_{N P} .
\end{gathered}
$$




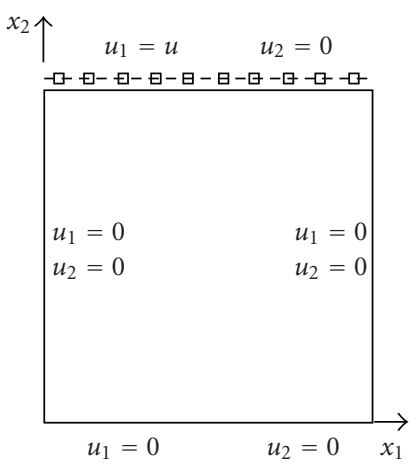

(a)

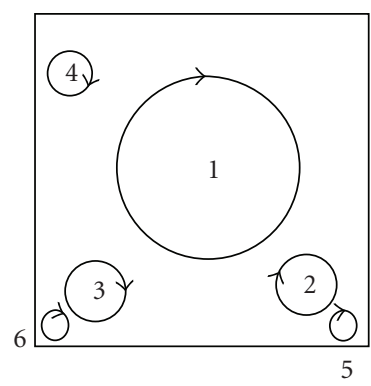

(b)
FIgURE 4: Lid-driven cavity test, (a) boundary condition (b) arrangement of eddies.

Then, the pressure and velocity components are corrected as

$$
\begin{gathered}
P=P^{*}+P^{\prime}, \\
u(i, j)=u(i, j)+\frac{d y}{a_{P U}(i, j)}\left(P^{\prime}(i, j)-P^{\prime}(i+1, j)\right), \\
v(i, j)=v(i, j)+\frac{d x}{a_{P V}(i, j)}\left(P^{\prime}(i, j)-P^{\prime}(i, j+1)\right) .
\end{gathered}
$$

The procedure is continued by solving the second pressure correction equation, rectifying the pressure and velocity with pressure correction and repeating the stages until convergence.

\section{Model Validation}

In this research, the N-S equations are discretized using a finite volume method of second-order accuracy in space based on Eulerian description at each time step. The N-S solver is developed based on S.G and C.G with a LES model as turbulent.

To validate the model, some standard tests such as liddriven cavity, Channel flow and Backward-facing step are used.

5.1. Lid-Driven Cavity. The fluid flow is in a rectangular container which moves tangentially to itself and parallel to one of the side walls. Due to the simplicity of the cavity geometry, applying a numerical method on this flow problem in terms of coding is quite easy and straight forward. Despite its simple geometry, the driven cavity flow retains a rich fluid flow physics manifested by multiple counter rotating recirculation regions on the corners of the cavity depending on the Reynolds number.

The geometry of the problem consist of boundary condition of velocity and the successive of eddies which is shown in Figure 4.

A sensitivity analysis is performed on mesh size (see Figures 5 and 6). As seen in Figures 5 and 6 by increasing the mesh size, the results become independence of it. For example, for $\mathrm{Re}=100$ and 3200 and for $N X=N Y=80$ and 150, the results on C.G and S.G become independence of mesh size.

The model is then performed on S.G and C.G for various Reynolds numbers at a range of 100 to 10000 and results were compared with those of Ghia et al. [11] that is regarded as the true solution of this problem [12]. Figures 7, 8, 9, and 10 show the results for horizontal and vertical velocity components.

The streamlines of fluid flow in cavity on C.G and S.G are shown on Figure 11.

For $\mathrm{Re}=100$ the horizontal velocity at the bottom and top of the domain are zero and $1 \mathrm{~m} / \mathrm{s}$, respectively. The maximum negative velocity is $-0.2 \mathrm{~m} / \mathrm{s}$ occurring at the middle point of the domain. The maximum negative velocity is increased due to an increase in Reynolds number. The location of this maximum velocity is moved toward downstream. For example for $\operatorname{Re}=10000$, this velocity is increased from $-0.2 \mathrm{~m} / \mathrm{s}$ to $-0.42 \mathrm{~m} / \mathrm{s}$ and it is location is moved from middle point to $0.07 \mathrm{~m}$ above of bottom. So by increasing the Reynolds number, the horizontal velocity gradient near the bottom and top is increased (see Figure 9). The reason for increasing the velocity gradient at two sides of the domain is that the thickness of boundary layer decreased as the Reynolds number increased leading to an increase in flow turbulence.

For $\operatorname{Re}=100$ the vertical velocity at both sides of the domain is zero and maximum negative and positive velocities occurs at $0.25 \mathrm{~m}$ and $0.8 \mathrm{~m}$ above the bottom equal to $0.17 \mathrm{~m} / \mathrm{s}$ and $-0.24 \mathrm{~m} / \mathrm{s}$, respectively. By increasing the Reynolds number, the velocity gradient is increased at both sides of domain. For example at $\mathrm{Re}=10000$, the maximum positive and negative velocities are increased from $0.17 \mathrm{~m} / \mathrm{s}$ to $0.4 \mathrm{~m} / \mathrm{s}$ and from $-0.24 \mathrm{~m} / \mathrm{s}$ to $-0.62 \mathrm{~m} / \mathrm{s}$, respectively. So by increasing the Reynolds number, the velocity gradient of vertical velocity at both sides is increased. The reason for increasing the vertical velocity gradient is the same as that of horizontal velocity gradient.

Figures 7 to 10 show that the horizontal and vertical velocities on low Reynolds number on C.G and S.G are predicted accurately (e.g., for $\mathrm{Re}=100$ ).

However increasing the Reynolds number increases the inconsistency between results so that the results of S.G are more close to Ghia results. These differences in the upstream and downstream of the domain are greater. While in the middle point, the results of C.G and S.G at various Reynolds numbers are predicted accurately.

Figure 11 illustrates the successive of eddies that are similar on C.G and S.G.

For low Reynolds number the stream lines on C.G and S.G are similar. However as the Reynolds number increases, the shape and the number of eddies change. For example at $\mathrm{Re}=10000$ there is an eddy at the bottom corners of domain on S.G while there are two eddies at the same locations on C.G.

5.2. Channel Flow. In this section, the solution of the channel flow for a long channel with a fully developed laminar flow is presented. The length and height of the channel are selected as $L=40 \mathrm{~m}$ and $h=1 \mathrm{~m}$ and the velocity in the entrance 


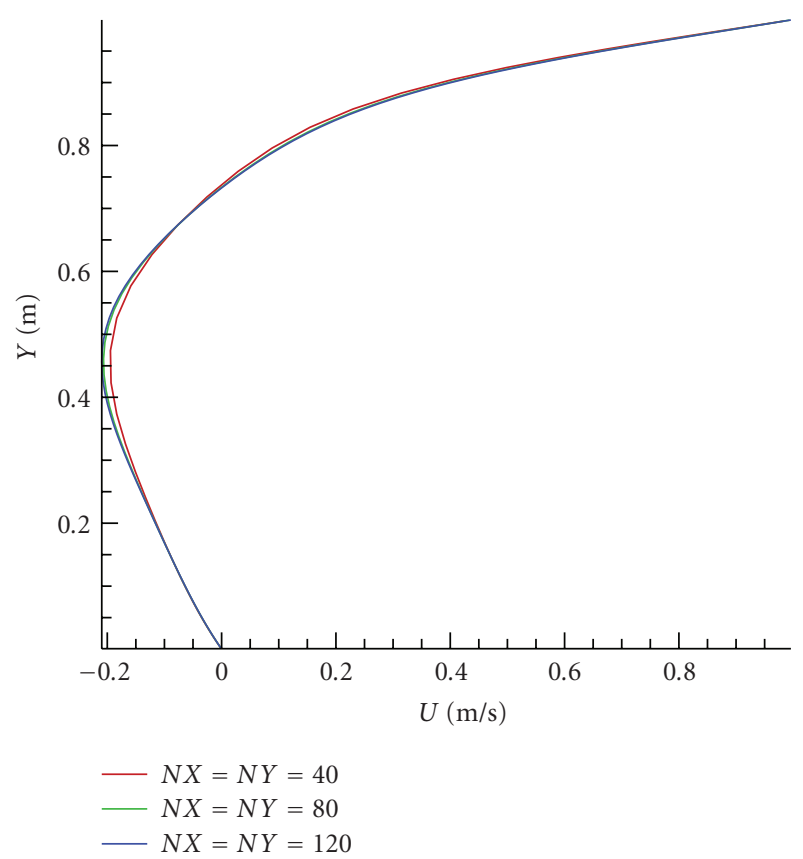

(a)

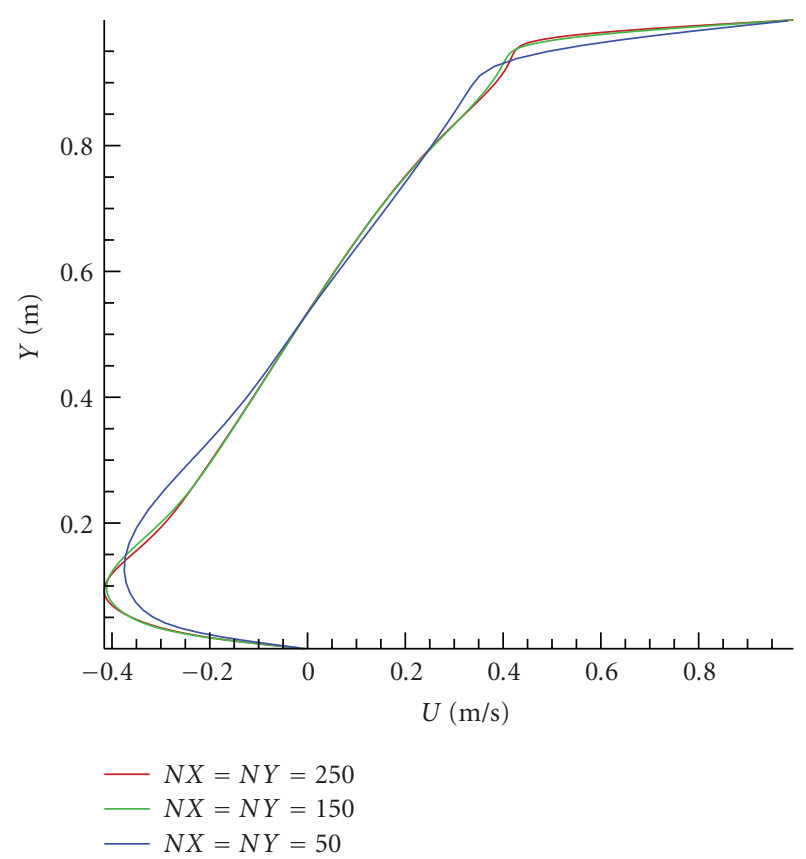

(b)

Figure 5: Mesh independency on S.G for (a) $\mathrm{Re}=100$ and (b) $\mathrm{Re}=3200$.

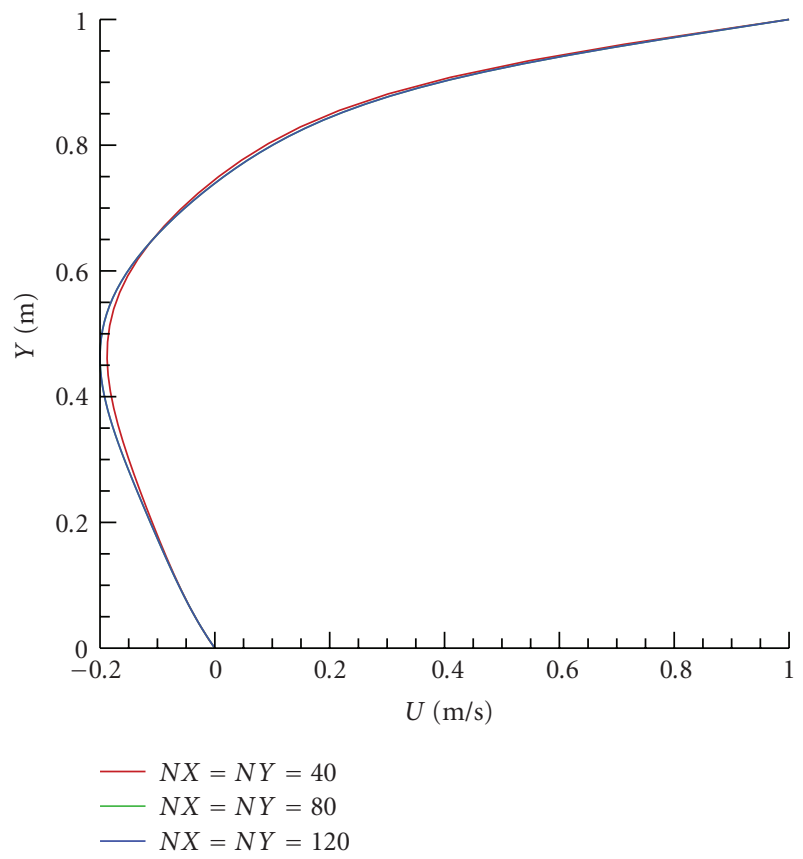

(a)

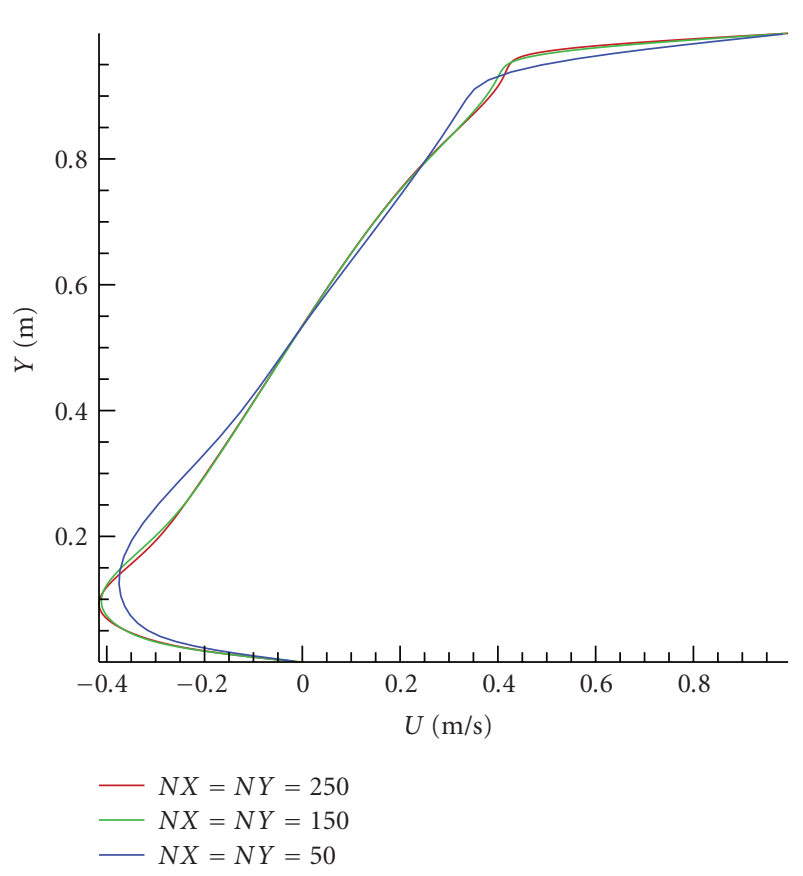

(b)

Figure 6: Mesh independency on C.G for (a) $\operatorname{Re}=100$ and (b) $\operatorname{Re}=3200$.

of the channel is $u=1 \mathrm{~m} / \mathrm{s}$. The velocity at the end of the channel is calculated on C.G and S.G at various Reynolds numbers in a range of 100 to 1000 . Figure 12 compares the results of C.G and S.G. It is evident that a very accurate result in comparison to analytical solution which is $u=6\left(Y-Y^{2}\right)$ is obtained.
The extension of boundary layer on C.G and S.G at $X=$ $(0.2-0.8) * L$ in Figure 13 shows that the results have nearly the same accuracy.

5.3. Flow over a Backward-Facing Step. To assess the accuracy of numerical method, the flow over a backward-facing step 


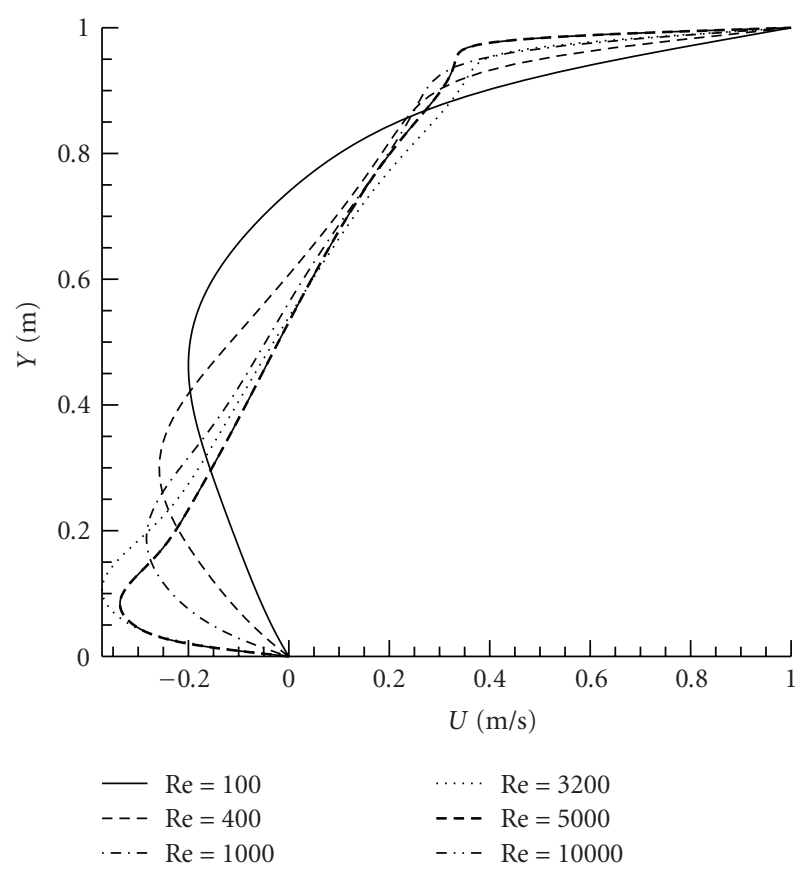

(a)

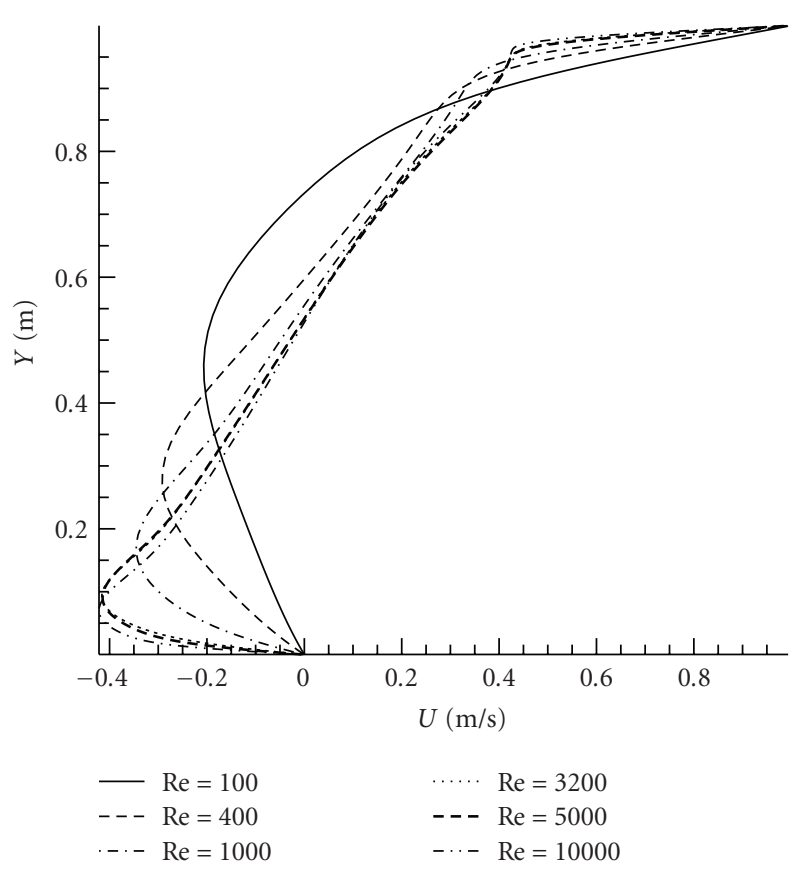

(b)

Figure 7: Comparison of horizontal velocity at various Reynolds numbers (a) C.G and (b) S.G.

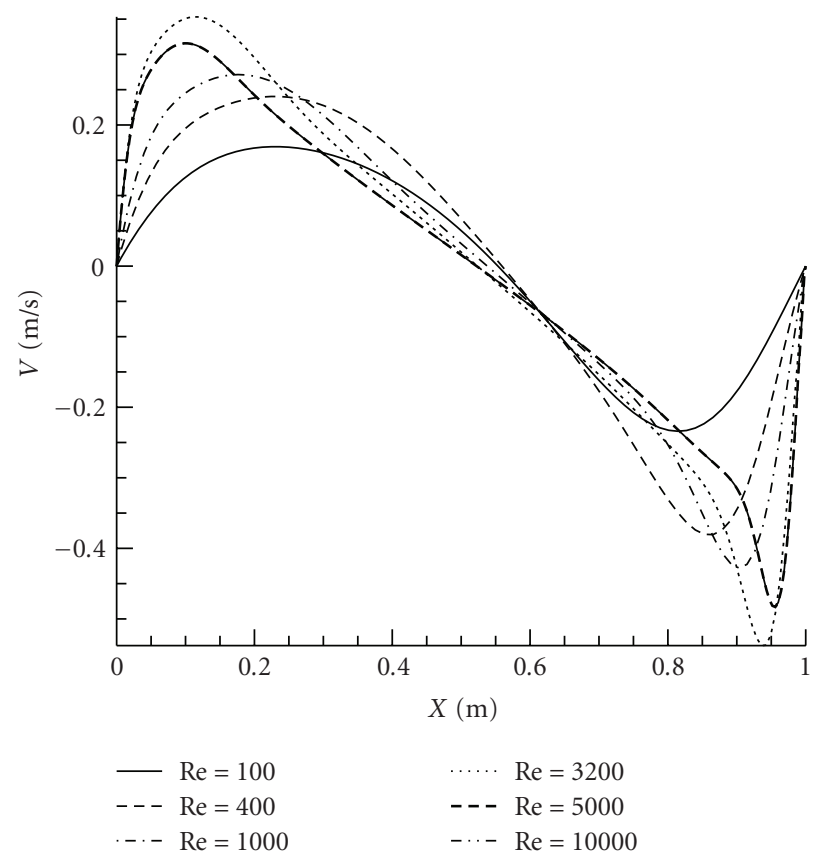

(a)

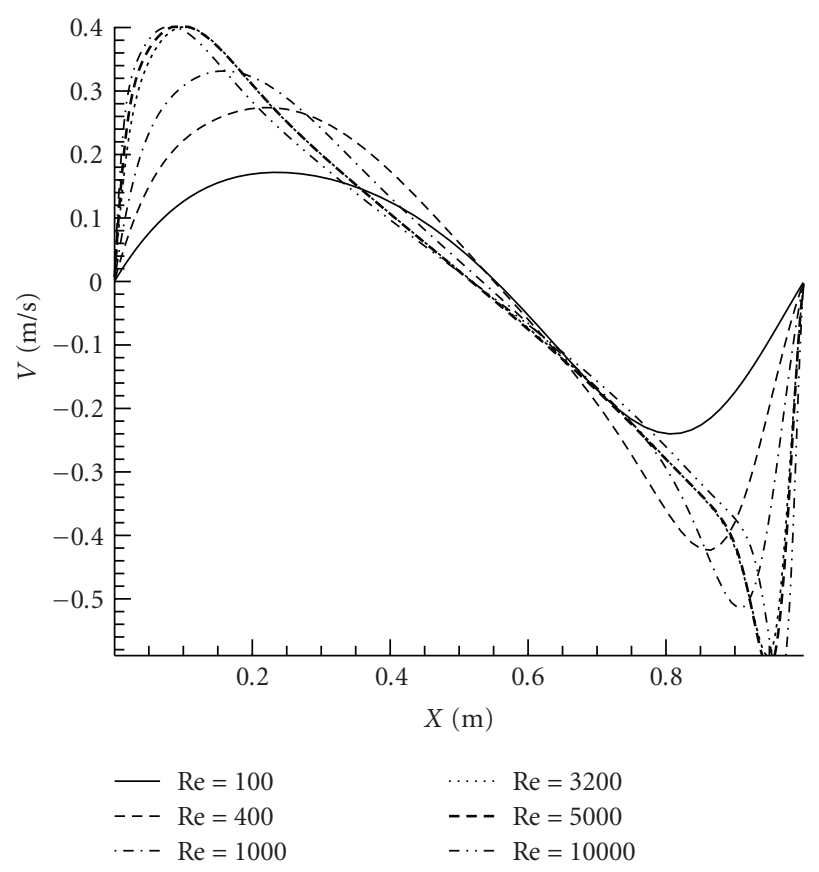

(b)

FIGURE 8: Comparison of vertical velocity at various Reynolds numbers (a) C.G and (b) S.G.

in a channel is a good test case. A dissipative scheme cannot predict the correct reattachment length of the recirculation zone downstream of the step [13]. The flow expands downstream of the step and reattaches on $X_{r}$ coordinate. A two-dimensional schematic of the backward-facing step is shown in Figure 14. In this figure, $d$ is the height of the channel; $h$ is the height of the step and $X_{r}$ is the reattachment length. In current work $d$ and $h$ selected as $1 \mathrm{~m}$ and $0.5 \mathrm{~m}$, respectively.

The regime of fluid flow on Backward facing step, for $\operatorname{Re}<400,400<\operatorname{Re}<3400$, and $\operatorname{Re}>3400$ is laminar, transient and turbulent, respectively [7]. For Re $<1000$ there 


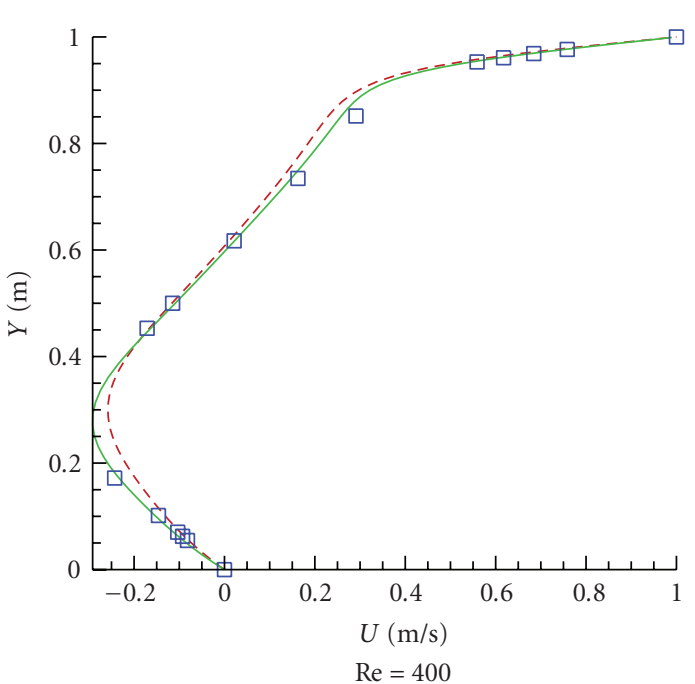

(a)

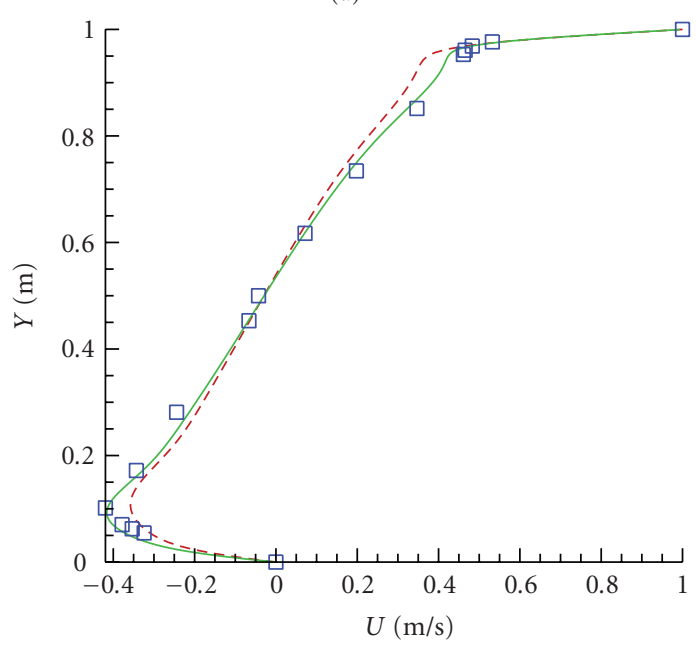

$\operatorname{Re}=3200$

(c)

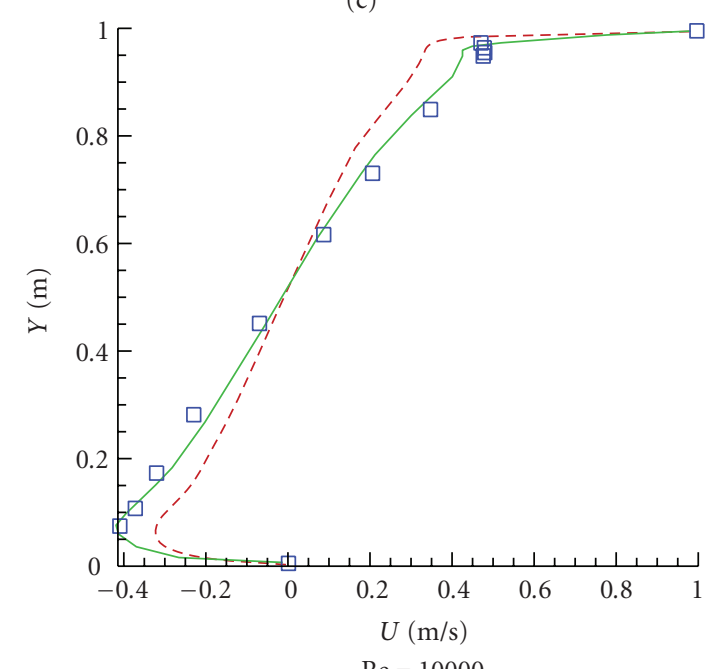

$\operatorname{Re}=10000$

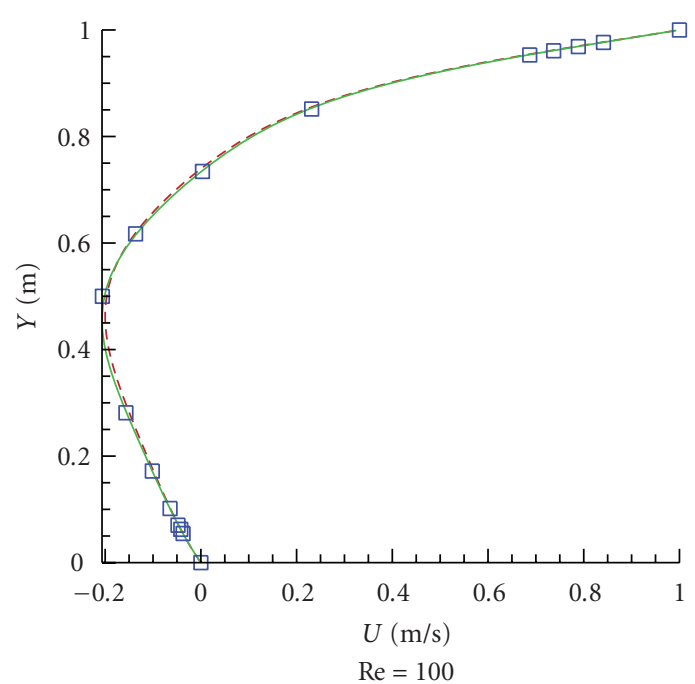

(b)

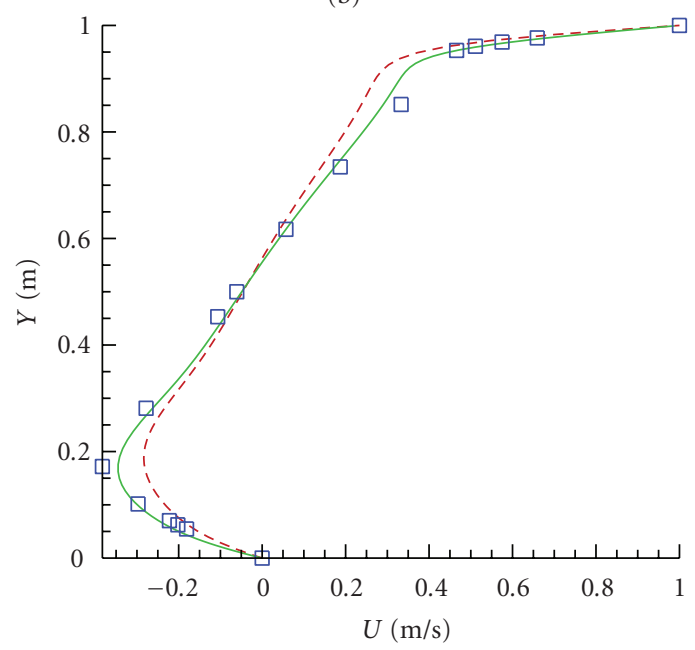

$\mathrm{Re}=1000$

(d)

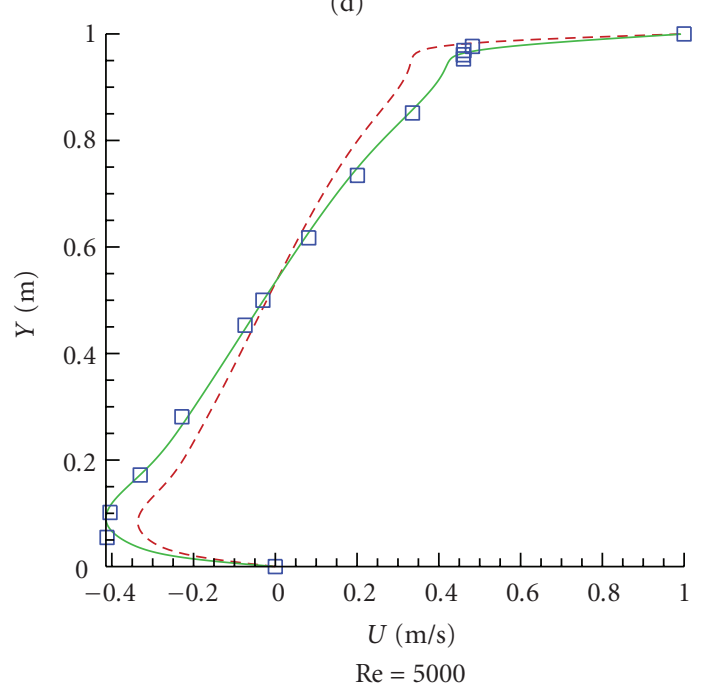

- - Collocated grid

— Staggered grid

$\square \quad$ Ghia et al.

(e)

(f)

Figure 9: Comparison of horizontal velocity on C.G and S.G and Ghia et al. results. 


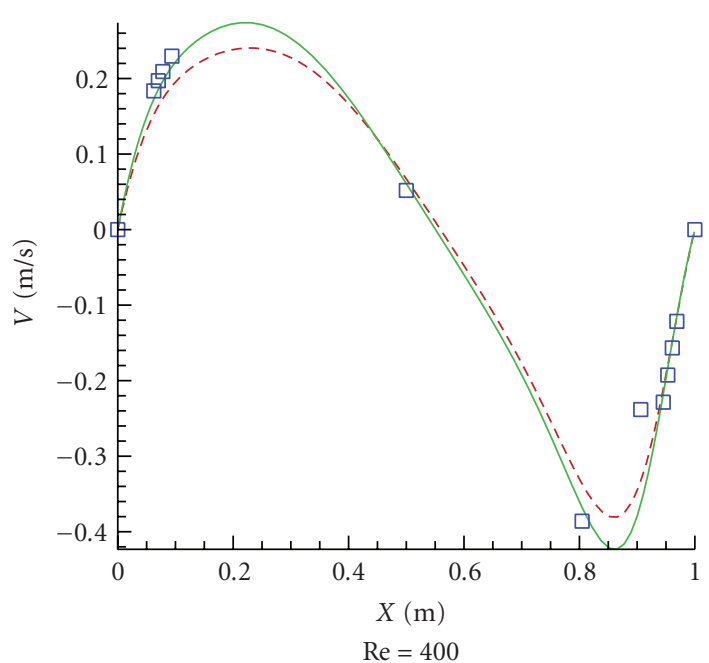

(a)

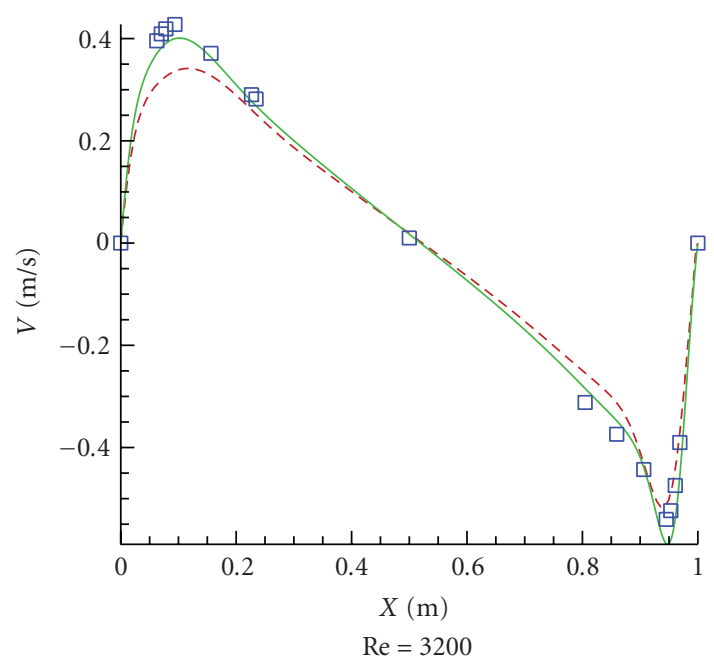

(c)

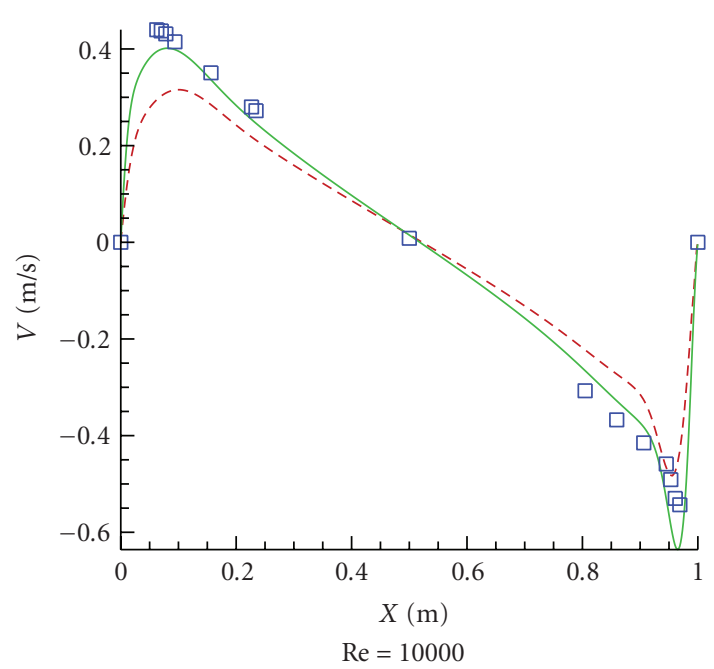

- - Collocated grid

- Staggered grid

$\square$ Ghia et al.

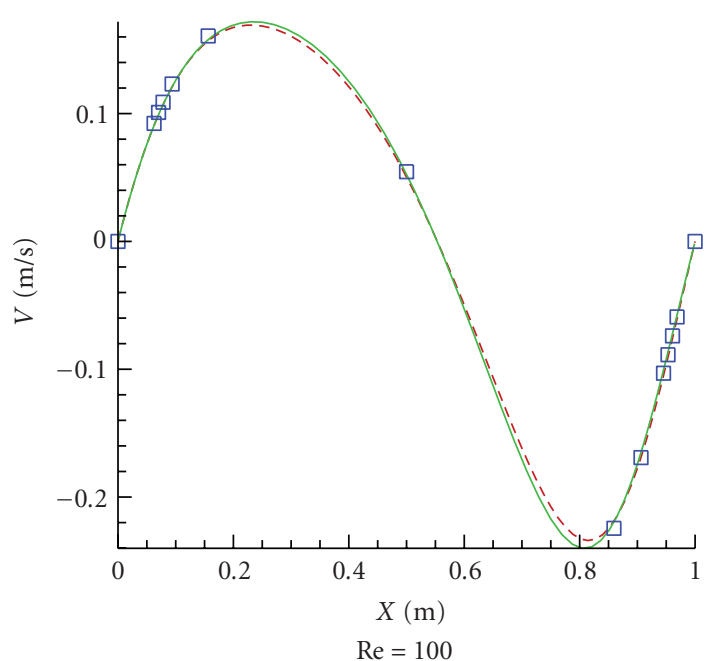

(b)

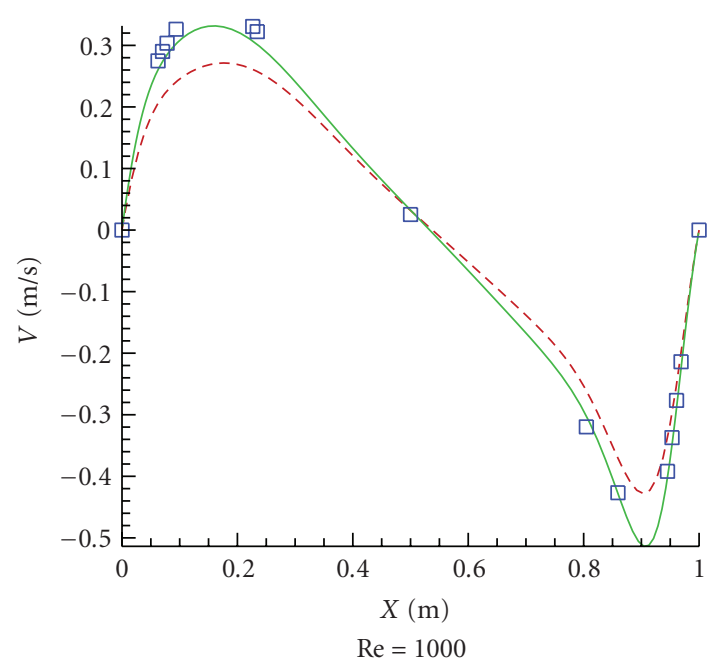

(d)

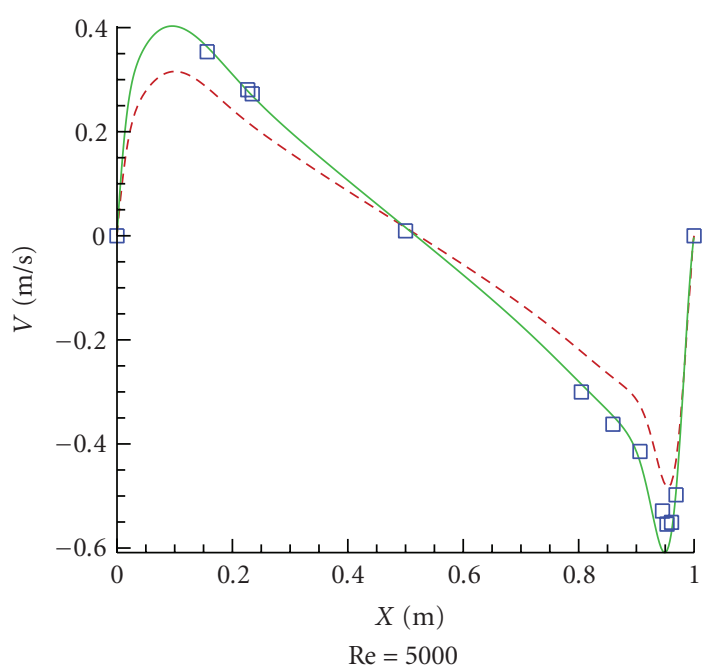

- - Collocated grid

- Staggered grid

$\square \quad$ Ghia et al.

(e)

(f)

Figure 10: Comparison of vertical velocity on C.G and S.G and Ghia et al. results. 

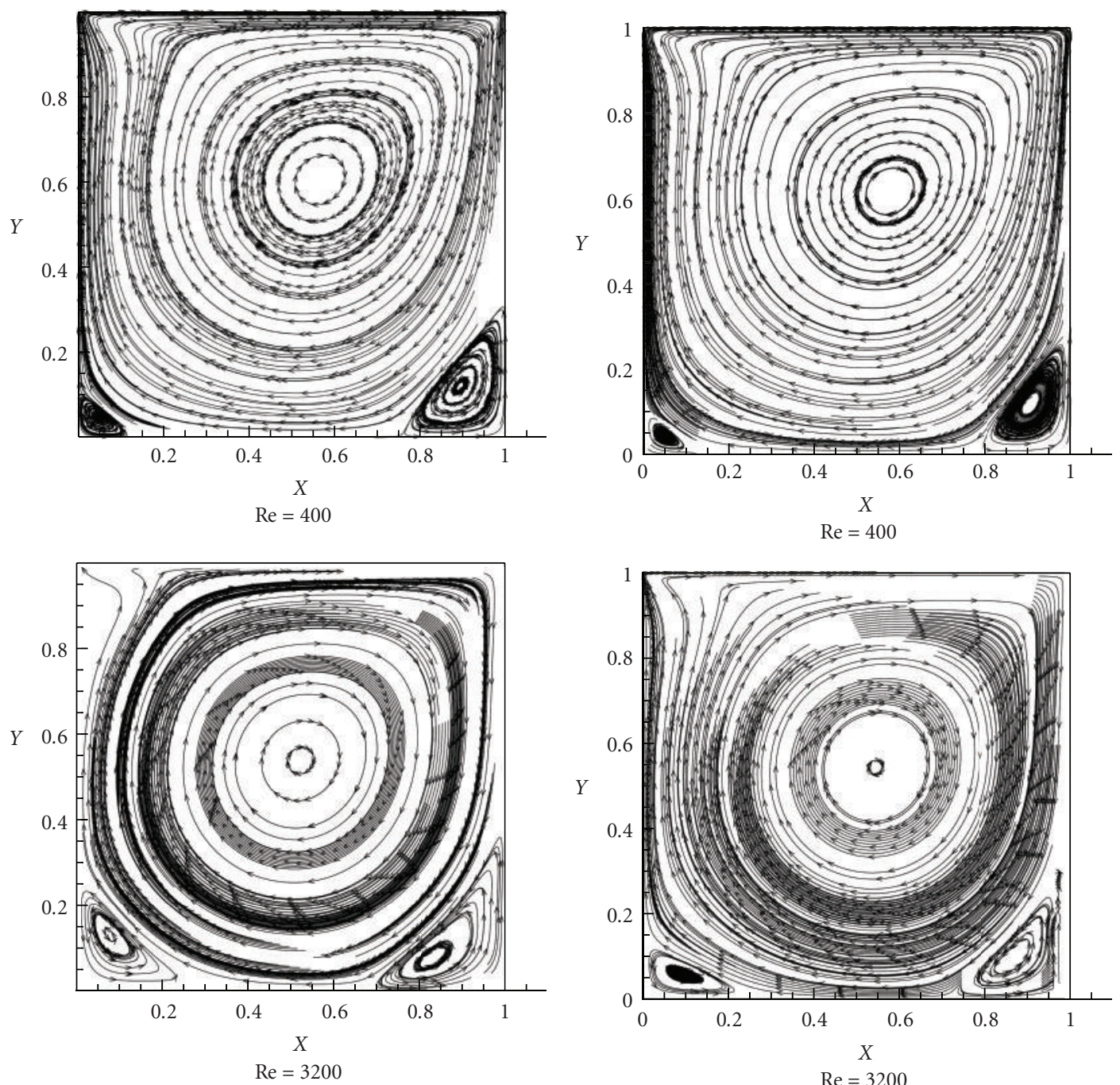

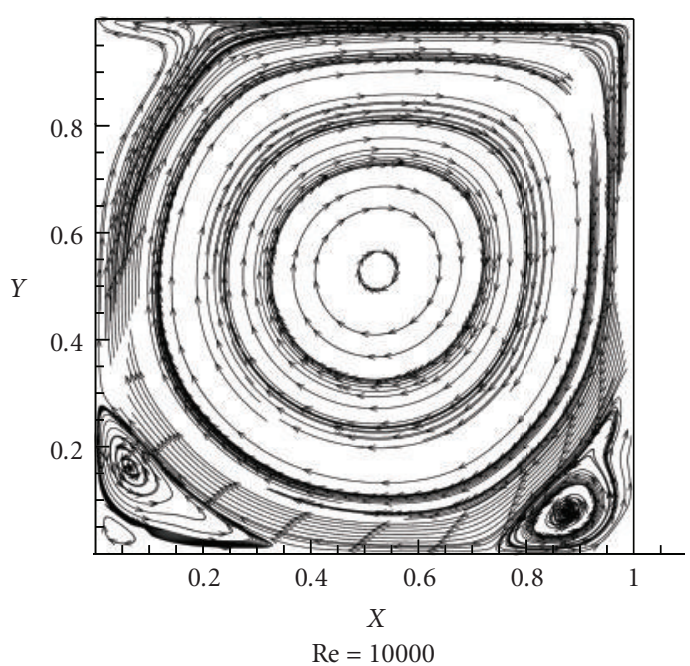

(a)

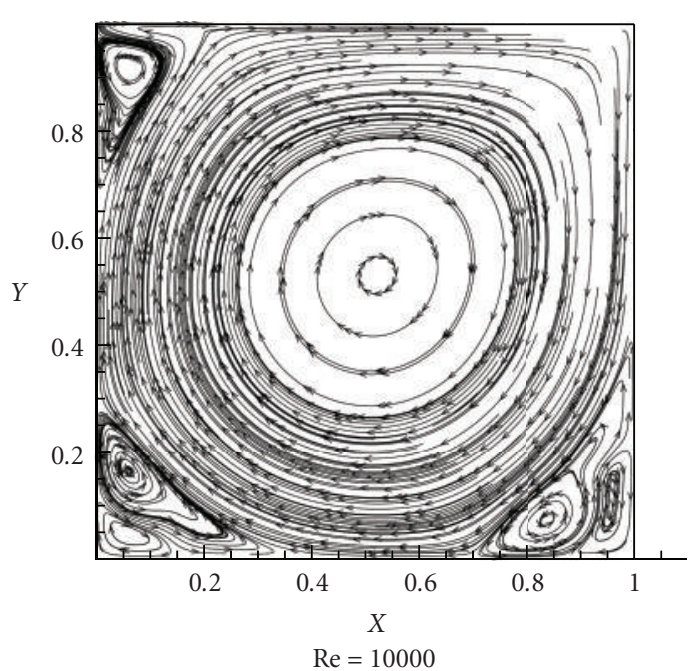

(b)

FIGURE 11: Comparison of stream lines for various Reynolds numbers (a) S.G and (b) C.G. 


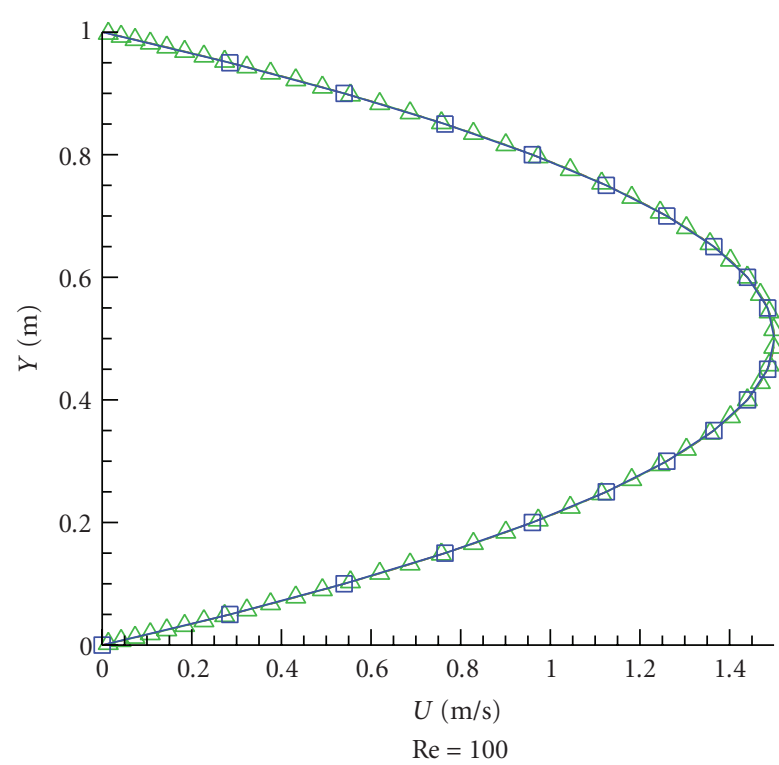

(a)

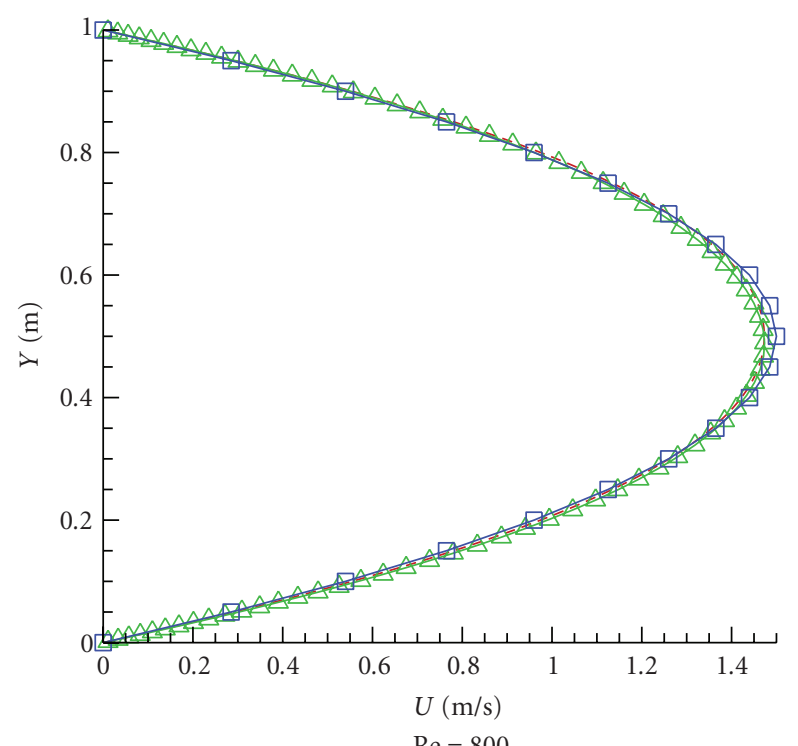

- - Collocated grid

$\triangle$ Staggered grid

$\square$ Analytical solution

(c)

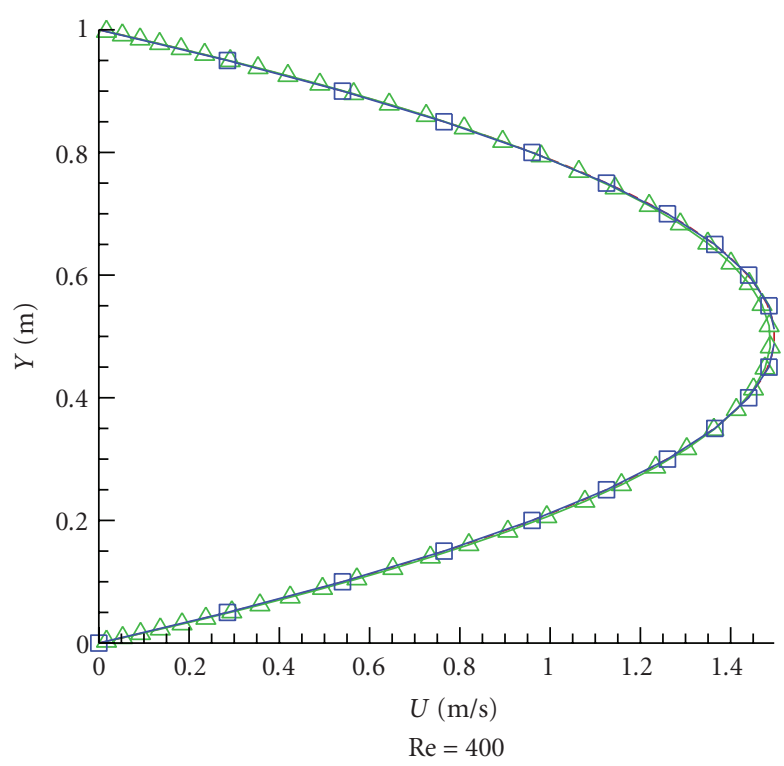

(b)

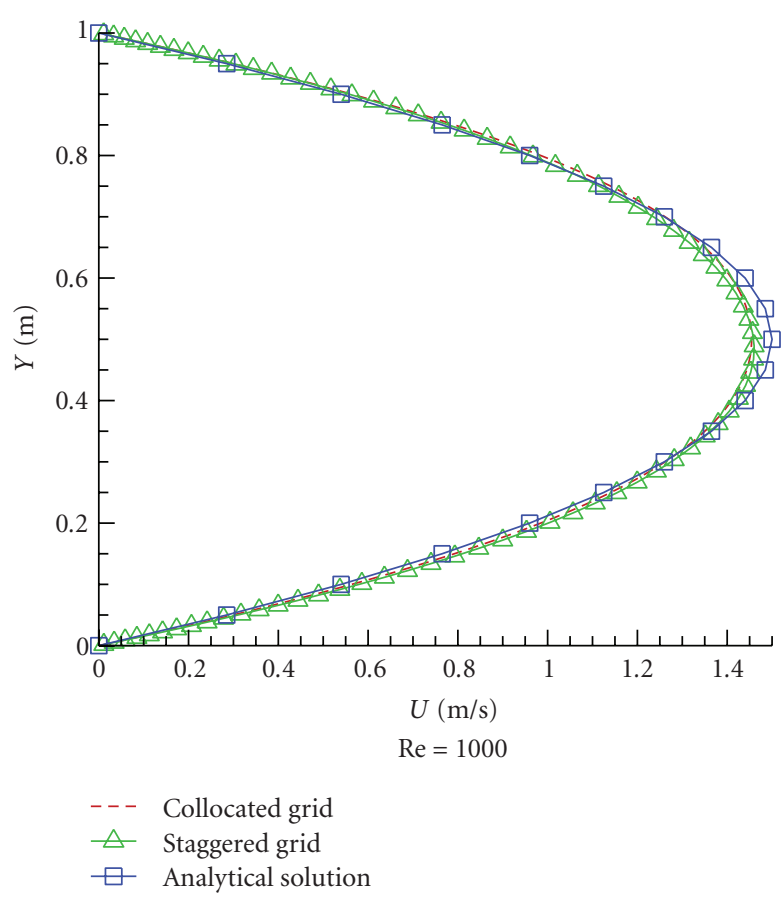

(d)

FIGURE 12: Channel test, comparison of fully developed profile on C.G and S.G and Analytical solution.

is an eddy in the downstream. But as the Reynolds number is increased, secondary or roof eddy is created in the upstream of the step and therefore the reattachment length is decreased [14].

In this section, the governing equations are solved and reattachment length at various Reynolds numbers on C.G and S.G is calculated. Finally the numerical and Armaly et al. results [8] are compared. Figure 15. shows this comparison. Armaly et al. results are regarded as the true solution of this problem.

It is evident from this figure that the results of S.G are better than C.G. But this result is not accurate for all the
Reynolds numbers. For example for $\operatorname{Re}=5000$, the result of S.G is very accurate but for $\mathrm{Re}=4000$ the result of S.G and C.G are not consistent with Almary et al. results.

The reattachment length of the recirculation zone downstream of the step is decreased for Reynolds numbers more than 1000. This is because of the existence of second eddy at the top of step (see Figure 16).

\section{Discussion and Conclusions}

In this paper, three test cases as lid-driven cavity, channel flow and backward-facing step are considered to compare the 


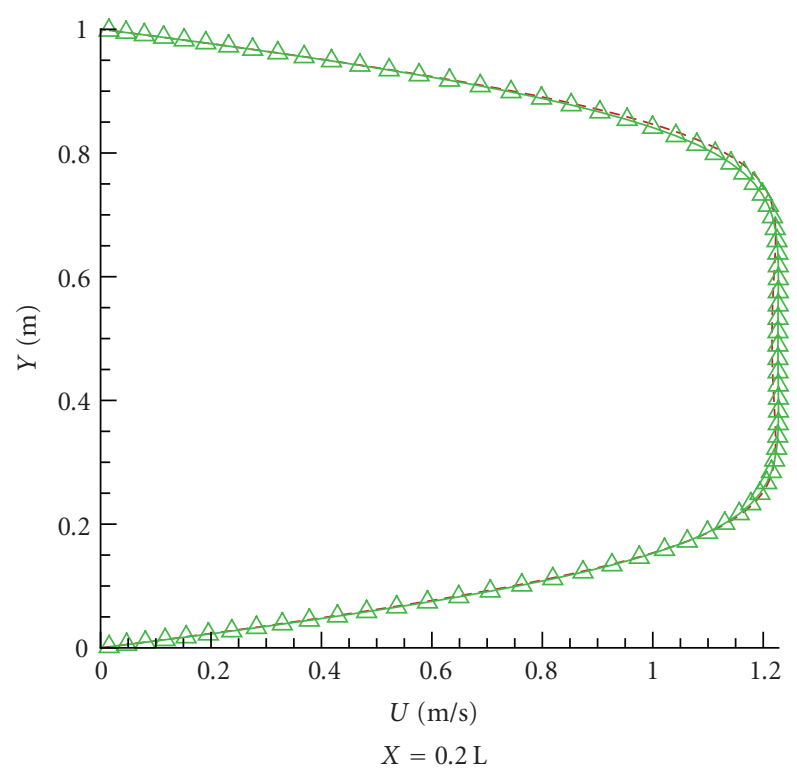

(a)

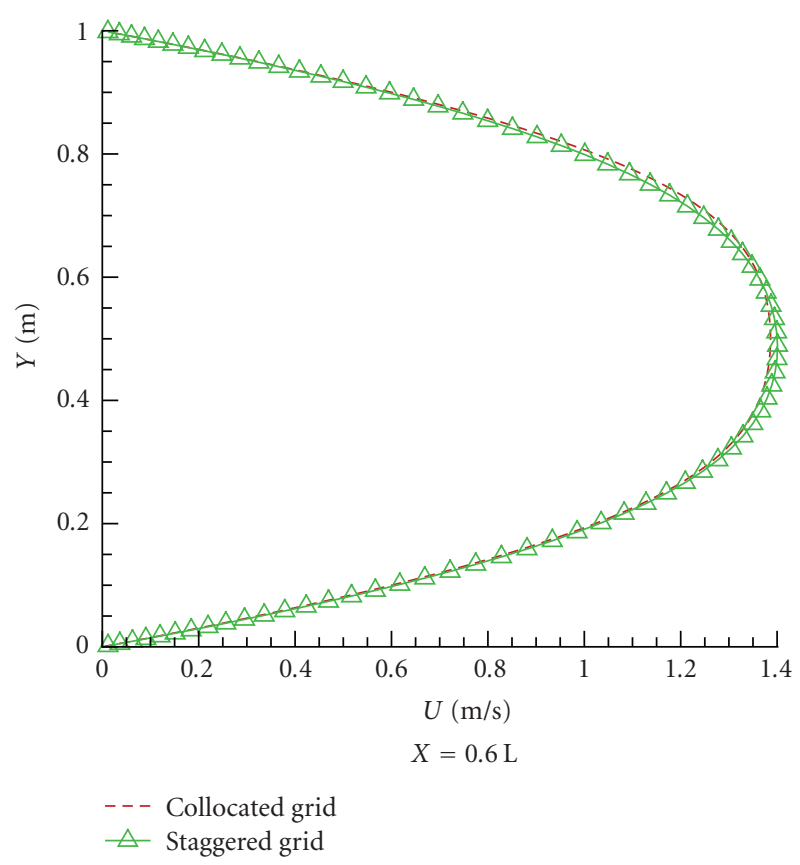

(c)

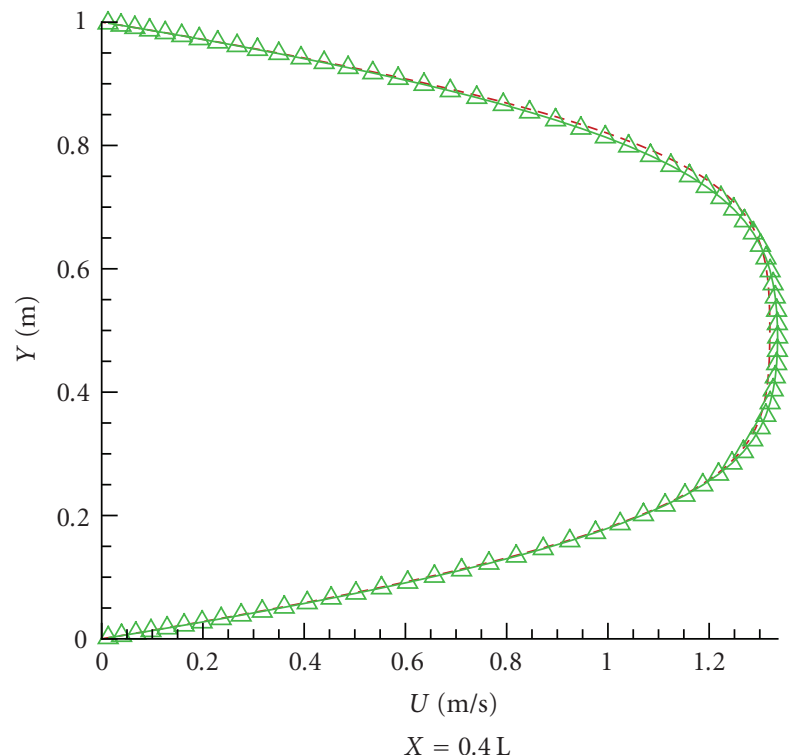

(b)

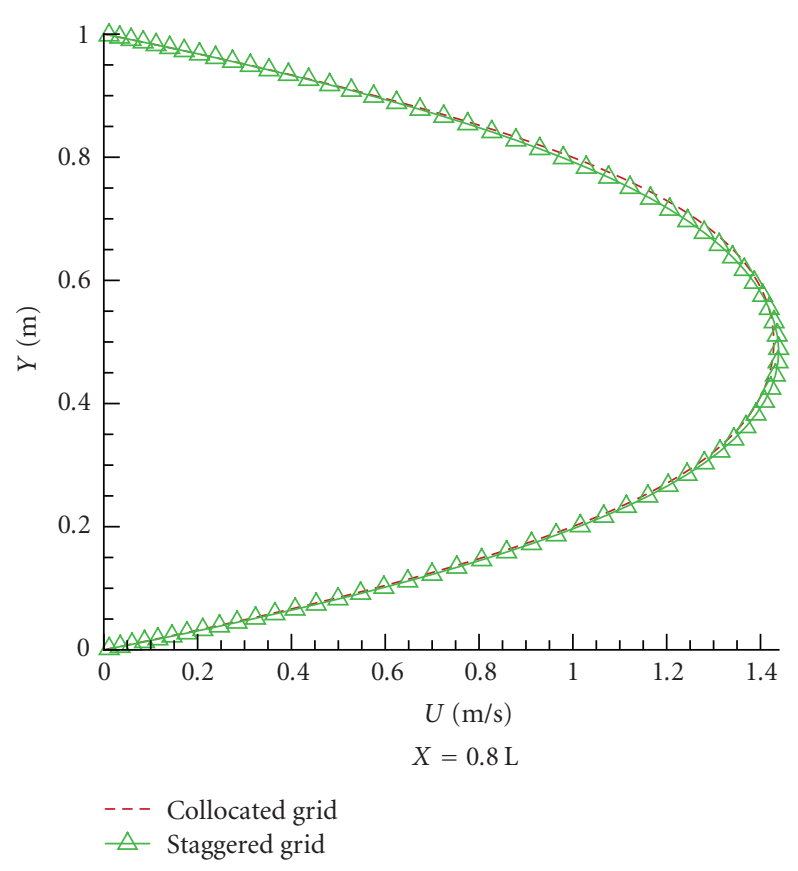

(d)

FIGURE 13: Channel test, development of Boundary layer on C.G and S.G.

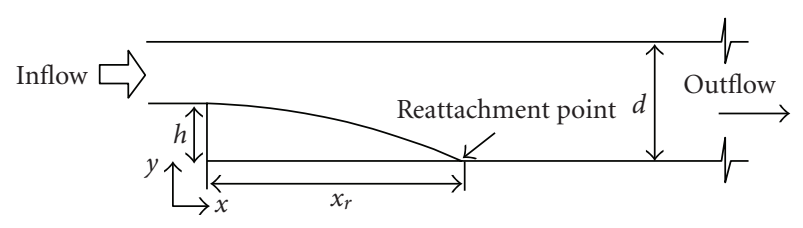

Figure 14: Geometry of step test.

accuracy of S.G and C.G methods. In the light of different results for N-S solver, following conclusion can be drawn:
In lid-driven cavity test, for low Reynolds numbers the results of S.G and C.G methods are nearly the same. Nevertheless for high Reynolds numbers, the results of S.G demonstrate better agreement. In lid-driven cavity test, for low Reynolds number the stream lines on C.G and S.G are similar. However as the Reynolds number increases, the shape and the number of eddies change. In the channel test, two grids present very good results for fully development and the extension of boundary layer. In backward-facing step, the reattachment length of the recirculation zone downstream of the step is decreased for Reynolds number more than 1000 . 


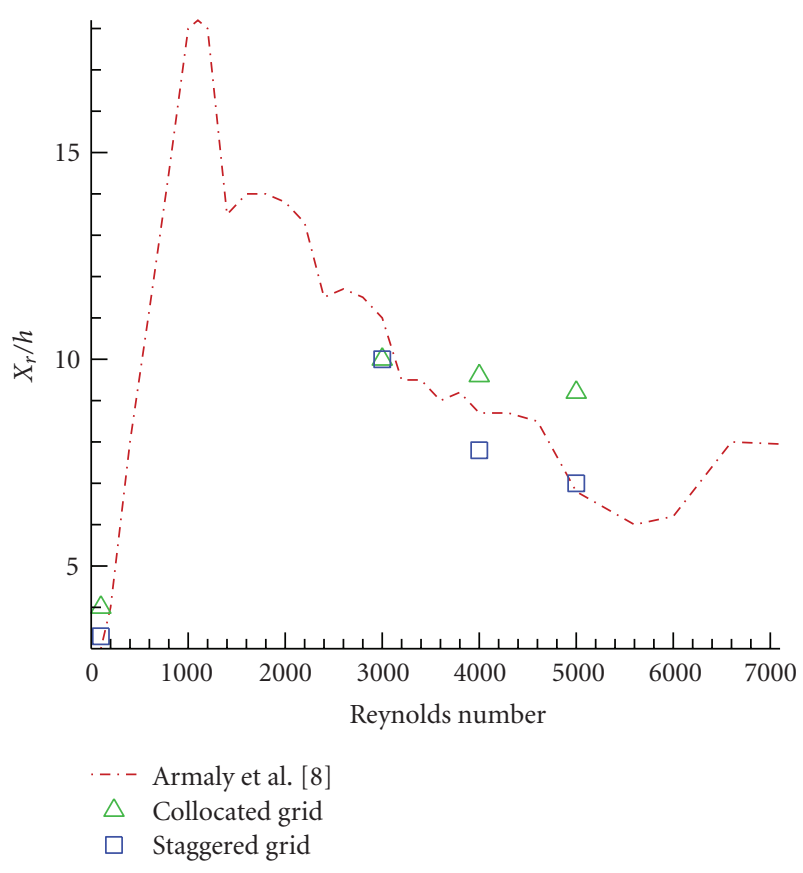

FIGURE 15: Comparison of Reattachment point on C.G and S.G and Armaly result.

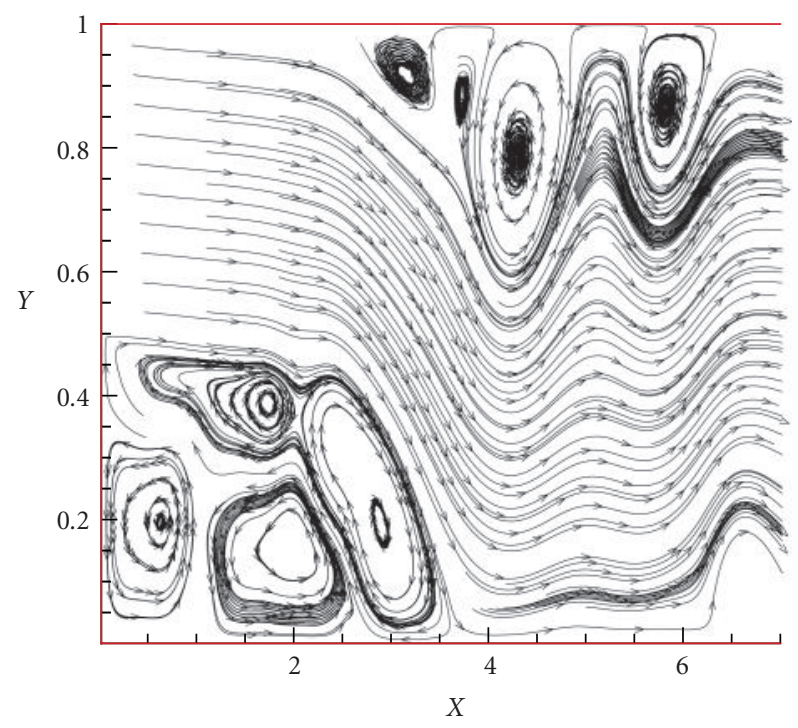

FIGURE 16: Step test, existence of second eddy at the top of step for $\operatorname{Re}>1000$.

This is because of the existence of second eddy at the top of step. In backward-facing step, the results of S.G are better than C.G.

It can be concluded from these test cases that, for problems such as lid-driven cavity and backward-facing step associated with eddy on the fluid flow, application of S.G leads to more accurate results. However for other problems such as channel flow, both methods nearly have the same accuracy.

\section{Nomenclature}

$C_{s}:$ A constant in the range $[0.025,0.65]$

$P$ : Static pressure

$\bar{S}_{i, j}:$ Strain rate tensor

$S_{\phi}$ : Source term in the $\phi$ direction

$t: \quad$ Time

$\bar{u}_{i} \quad$ Filtered velocity in $x$ direction

$\bar{u}_{j}$ : Filtered velocity in $y$ direction

$u$ : Velocity components in $x$ direction

$v$ : Velocity components in $y$ direction

$V$ : Velocity vector.

\section{Greek Symbol}

$\Delta:$ Length of filter

$\Delta_{x}$ : Mesh size in $x$ direction

$\Delta_{y}:$ Mesh size in $y$ direction

$\Delta_{z}$ : Mesh size in $z$ direction

$v_{t}$ : Turbulence eddy viscosity

$\mu$ : Dynamic fluid viscosity

$\rho$ : Fluid density.

\section{References}

[1] H. K. Versteeg and W. Malalaskera, An Introduction to Computational Fluid Dynamics, The Finite Volume Method, Longman, Harlow, UK, 1995.

[2] U. Piomelli, "Large-eddy simulation: achievements and challenges," Progress in Aerospace Sciences, vol. 35, no. 4, pp. 335362, 1999.

[3] J. H. Ferziger and M. Peric, Computational Methods for Fluid Dynamics, Springer, 3rd edition, 2002.

[4] A. K. Prasad and J. R. Koseff, "Reynolds number and end-wall effects on a lid-driven cavity flow," Physics of Fluids, vol. 1, no. 2, pp. 208-218, 1989.

[5] A. Gokarn, F. Battaglia, R. O. Fox, J. C. Hill, and J. Reveillon, "Large eddy simulations of incompressible turbulent flows using parallel computing techniques," International Journal for Numerical Methods in Fluids, vol. 56, no. 10, pp. 1819-1843, 2008.

[6] J. Kim, P. Moin, and R. Moser, "Turbulence statistics in fully developed channel flow at low Reynolds number," Journal of Fluid Mechanics, vol. 177, pp. 133-166, 1987.

[7] J. H. Nie and B. F. Armaly, "Reverse flow regions in threedimensional backward-facing step flow," International Journal of Heat and Mass Transfer, vol. 47, no. 22, pp. 4713-4720, 2004.

[8] B. F. Armaly, F. Durst, J. C. F. Pereira, and B. Schoenung, "Experimental and theoretical investigation of backwardfacing step flow," International Journal of Fluid Mechanics, vol. 127, pp. 473-496, 1983.

[9] J. Smagorinsky, "General circulation experiments with the primitive equations," Monthly Weather Review, vol. 91, no. 3, pp. 99-164, 1963.

[10] L. Davidson, “Turbulence Modeling, Large Eddy Simulation,” 2006.

[11] U. Ghia, K. N. Ghia, and C. T. Shin, "High-Re solutions for incompressible flow using the navier-stokes equations and a multigrid method," Journal of Computational Physics, vol. 48, no. 3, pp. 387-411, 1982. 
[12] J. R. Koseff and R. L. Street, "On end wall effects in a lid-driven cavity flow," ASME, Journal of Fluids Engineering, vol. 106, no. 4, pp. 385-389, 1984.

[13] V. M. G. Molla, J. L. M. González, and L. L. Zamora, "Use of high accuracy schemes to handle free surfaces in computing unsteady two-phase flows," Computational Method Applied Mechanical Engineering, vol. 86, pp. 162-271, 1998.

[14] T. P. Chiang, W. H. Sheu, W. H. Sheu, and C. C. Fang, "Numerical investigation of vortical evolution in a backwardfacing step expansion flow," Applied Mathematical Modeling, vol. 23, no. 12, pp. 915-932, 1999. 

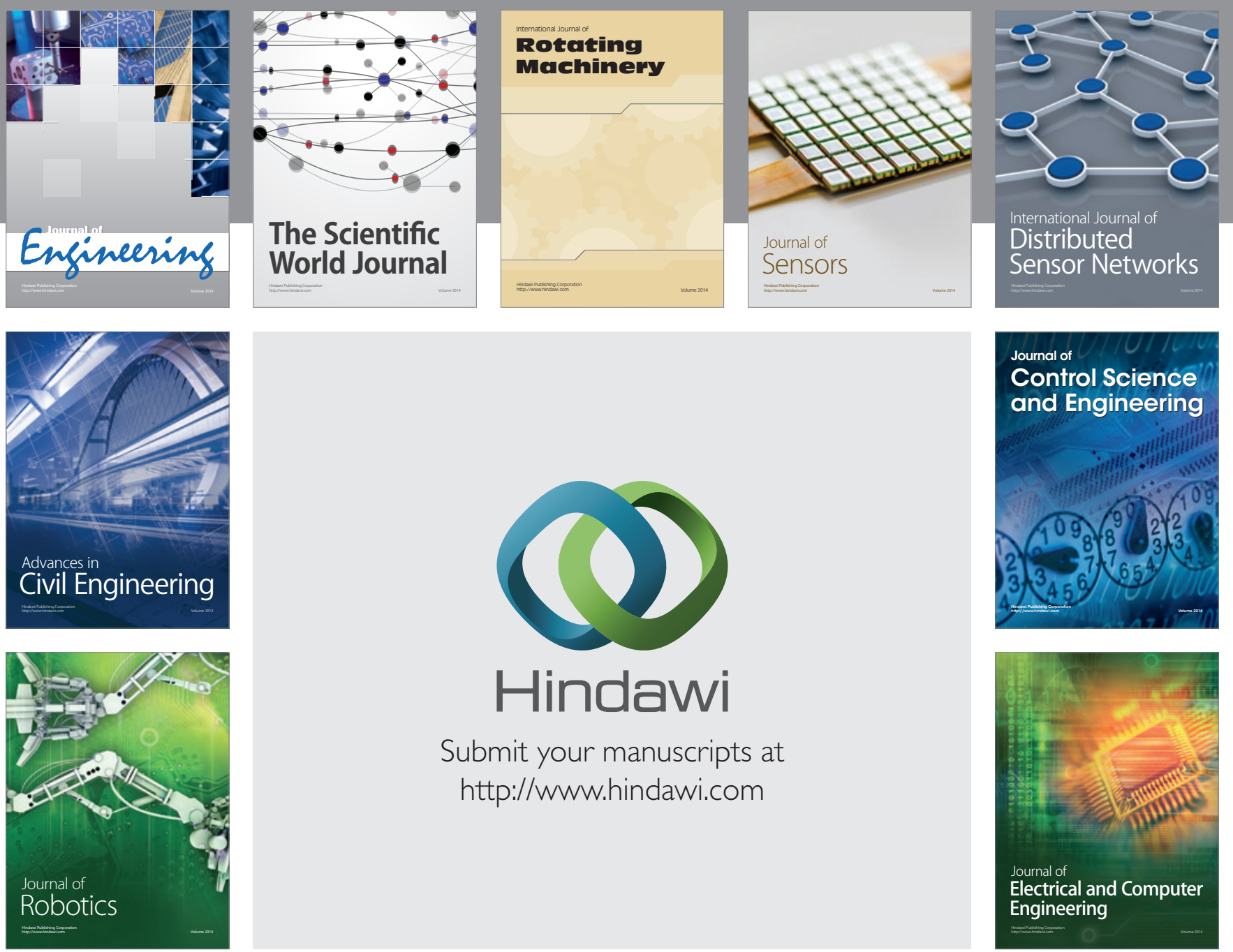

Submit your manuscripts at

http://www.hindawi.com
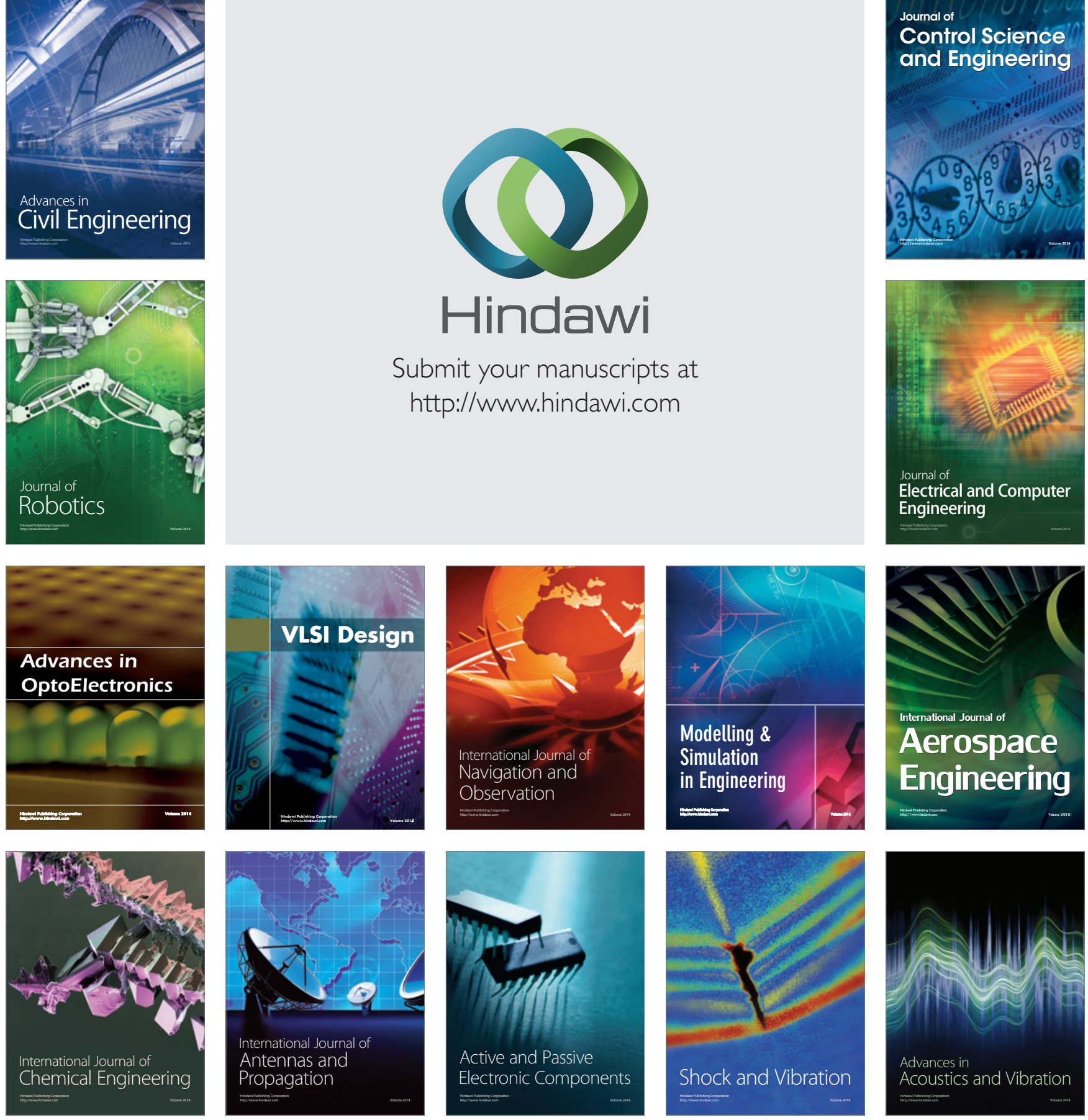